\title{
Chapter 14 \\ Enabling Intelligent Systems, Applications, and Analytics for Smart Environments Using Real-time Linked Dataspaces
}

Keywords Dataspaces · Intelligent systems · Energy management · Water management $\cdot$ Smart building $\cdot$ Smart airport $\cdot$ Smart home $\cdot$ Smart environments

\subsection{Introduction}

The design of next-generation smart environments poses significant technical challenges with data management, data integration, and real-time processing of dynamic data, and non-technical challenges such as engaging end-users and supporting cultural and organisational changes. Real-time Linked Dataspaces (RLD) are data platforms designed explicitly to tackle these challenges. In this chapter, we provide an overview of how a Real-time Linked Dataspace enables the creation of intelligent systems within several smart environments to demonstrate and evaluate their effectiveness.

This chapter is structured as follows; it begins in Sect. 14.2 with a discussion of the challenges to delivering intelligent energy and water management systems. Section 14.3 introduces Real-time Linked Dataspaces. Section 14.4 details the real-world pilots where the dataspace approach was deployed and evaluated, including an overview of the different user groups involved in each pilot. Section 14.5 details the different types of intelligent applications that were developed using the dataspace, and the chapter concludes in Sect. 14.6.

\subsection{Intelligent Energy and Water Management}

There is a significant opportunity to accelerate the use of intelligent systems to improve energy and water resource management and conservation by analysing, designing, and implementing intelligent systems to increase demand and supply 
efficiency. To manage energy and water holistically within a smart environment, it is essential to use decision support tools that present meaningful and contextual information about usage, price, and availability of energy and water intuitively and interactively to users. Users will need different forms of information to manage their energy and water consumption, from home users managing their personal water usage, business users managing the water consumption of their commercial activities, to municipalities managing regional distribution and consumption at the level of a city or a region. To develop intelligent applications for these diverse users, it is necessary to leverage knowledge from several different domains including metering (household, neighbourhood), water collection and catchment management, energy generation, environmental impacts, water quality, distribution networks, end-user feedback, occupancy patterns, and meteorological data. The design of nextgeneration intelligent energy and water management systems poses significant technical challenges with data management, data integration, and real-time processing of dynamic data, and non-technical challenges such as engaging end-users and supporting cultural and organisational changes (see Chap. 2). To support the interconnection of intelligent systems in the data ecosystem that surrounds a smart environment, there is a need to enable the sharing of data among systems.

\subsection{Real-time Linked Dataspaces}

A data platform can provide a clear framework to support the sharing of data among a group of intelligent systems within a smart environment [1] (see Chap. 2). In this book, we advocate the use of the dataspace paradigm within the design of data platforms to enable data ecosystems for intelligent systems.

A dataspace is an emerging approach to data management that recognises that in large-scale integration scenarios, involving thousands of data sources, it is difficult and expensive to obtain an upfront unifying schema across all sources [2]. Within dataspaces, datasets co-exist but are not necessarily fully integrated or homogeneous in their schematics and semantics. Instead, data is integrated on an as-needed basis with the labour-intensive aspects of data integration postponed until they are required. Dataspaces reduce the initial effort required to set up data integration by relying on automatic matching and mapping generation techniques. This results in a loosely integrated set of data sources. When tighter semantic integration is required, it can be achieved in an incremental pay-as-you-go fashion by detailed mappings among the required data sources.

We have created the Real-time Linked Dataspace (RLD) (see Chap. 4) as a data platform for intelligent systems within smart environments. The RLD combines the pay-as-you-go paradigm of dataspaces with linked data and real-time stream and event processing capabilities to support a large-scale distributed heterogeneous collection of streams, events, and data sources [4]. To validate the RLD approach, 
it has been used in the development of intelligent applications and decision support for five smart energy and water environments. The remainder of this chapter details these pilots.

\subsection{Smart Environment Pilot Deployments}

Over the past number of years, we have been involved in a number of projects $[18,62,63]$ concerned with investigating the use of a Real-time Linked Dataspace as a data platform for intelligent systems within smart environments, specifically targeting intelligent systems for smart energy and water management. As detailed in Fig. 14.1, the five pilot smart environments are Airport, Office, Home, Mixed Use and School.

\subsubsection{Smart Airport (Linate, Milan)}

Linate Airport represents large-scale commercial energy and water consumers with mixed water use from washing activities, toilets, restaurants, flight operations, to safety critical infrastructure for emergency response. The intelligent systems and applications in Linate target a variety of users in a business environment (including

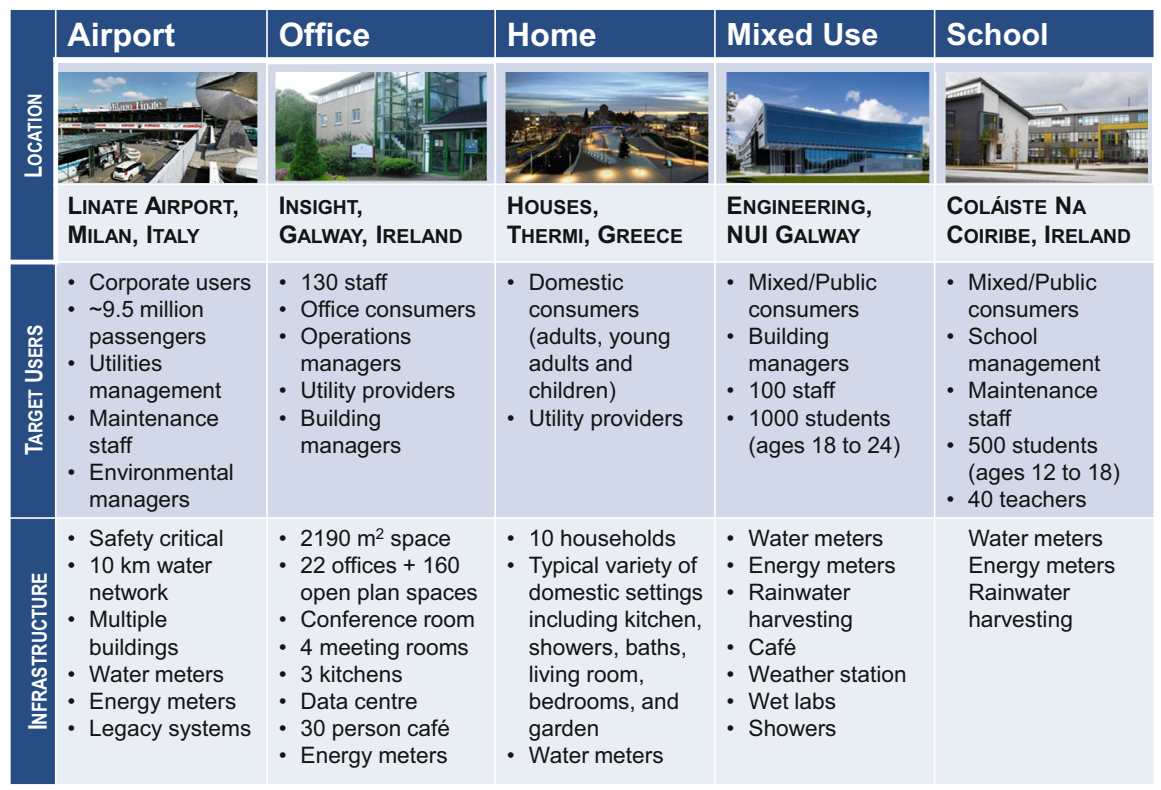

Fig. 14.1 Five smart environments [4] 
executives, operational managers, and technical staff), each one having a specific role in the company's structure. Apart from the company's employees, applications also target the airport passengers representing the public. Multiple legacy building management systems are in use across the different buildings. The variety of sensors used in the airport require the management of heterogeneous events and their availability to applications in near-real-time. Significant contextual data from the airport's operational systems are needed to process the events for decision-making, presenting significant challenges with legacy data integration and access control.

\subsubsection{Smart Office (Galway, Ireland)}

The Insight Building hosts approximately 130 staff in a dedicated building with $2190 \mathrm{~m}^{2}$ of space, comprising offices, open plan workspaces, a 90-seat conference room, four meeting rooms, three kitchens, one air-conditioned data centre, and a 30-person café. The building was built in the 1990s and did not employ a building management system or an energy management system. The building has been retrofitted with energy sensors and a simple energy management system. As typically in an organisation, Insight has several information systems that run its operations, including finance and enterprise resource planning, budgeting, and Office IT assets. These enterprise systems can help in identifying energy wastage and promote conservation actions.

\subsubsection{Smart Homes (Municipality of Thermi, Greece)}

The Municipality of Thermi in Greece provides a residential smart water pilot constituting a representative sample of ten domestic residences with different profiles. The target audience of smart home water applications are the resident adults and children. Other interested users include the municipality management and a developer community for smart home "Apps", research scientists, and the local water utility. Data from Internet of Things (IoT) devices in each home needs to be managed in a near-real-time manner to provide feedback to users on their water consumption. Sensor data needs to be enriched and linked to entities describing the households' water outlets. Securely sharing datasets with both the research and the developer community was an essential requirement.

\subsubsection{Mixed Use (Galway, Ireland)}

The Engineering Building at NUI Galway is a state-of-the-art smart building with large quantities of sensors and actuators for its management. This smart environment is designed to be a "living laboratory" where the building itself is an interactive 
teaching tool. The building includes lecture halls, classrooms, offices, laboratory facilities, café, showers, and bathrooms. Intelligent energy and water applications target staff members, managers, technicians, researchers, and students. As with the smart home example, the requirement to make data easily reusable by occupants in the environment is an important requirement. Also, staff members are interested in understanding usage behaviours and detecting saving opportunities, and students are interested in visualising the building consumption and utilising data from the environment in their projects and research works.

\subsubsection{Smart School (Galway, Ireland)}

Coláiste na Coiribe is an Irish language secondary school that has been constructed in 2015 at a suburban location in Galway City in Ireland. It includes classrooms, offices, sports halls, and associated toilet and shower facilities. The school accommodates students aged 12 to 18 years, together with teaching and operational staff. The school has been fitted with a commercial state-of-the-art building management system to manage its energy and water consumption. An essential requirement in this environment is to customise the communication of water and energy data for the diverse range of school stakeholders.

\subsubsection{Target Users Groups}

Smart environments can engage a wide range of end-users with different interests and priorities, from corporate managers looking to improve the performance of their business to school children who want to explore and learn more about sustainability and their effects on the environment. The target users across the five pilot smart environments (as illustrated in Fig. 14.2) are:

- Building Managers and Operations Staff: Managers, technicians, and engineers as well as other staff members with responsibility for the operations of buildings. These are adult users that have an advanced level of education and are highly skilled.

- Passengers: Passengers that range from business travellers to a variety of casual travellers from different age groups, from kids to adults.

- Corporate Staff: Office and administration staff that are found in a typical organisation. These adults are often highly educated and skilled (e.g. administration, HR, marketing, and sales), but not in the technical area of energy and water management.

- Families: Family units with potentially multiple generations in one home, including children, young adults, adults, parents, and grandparents. 


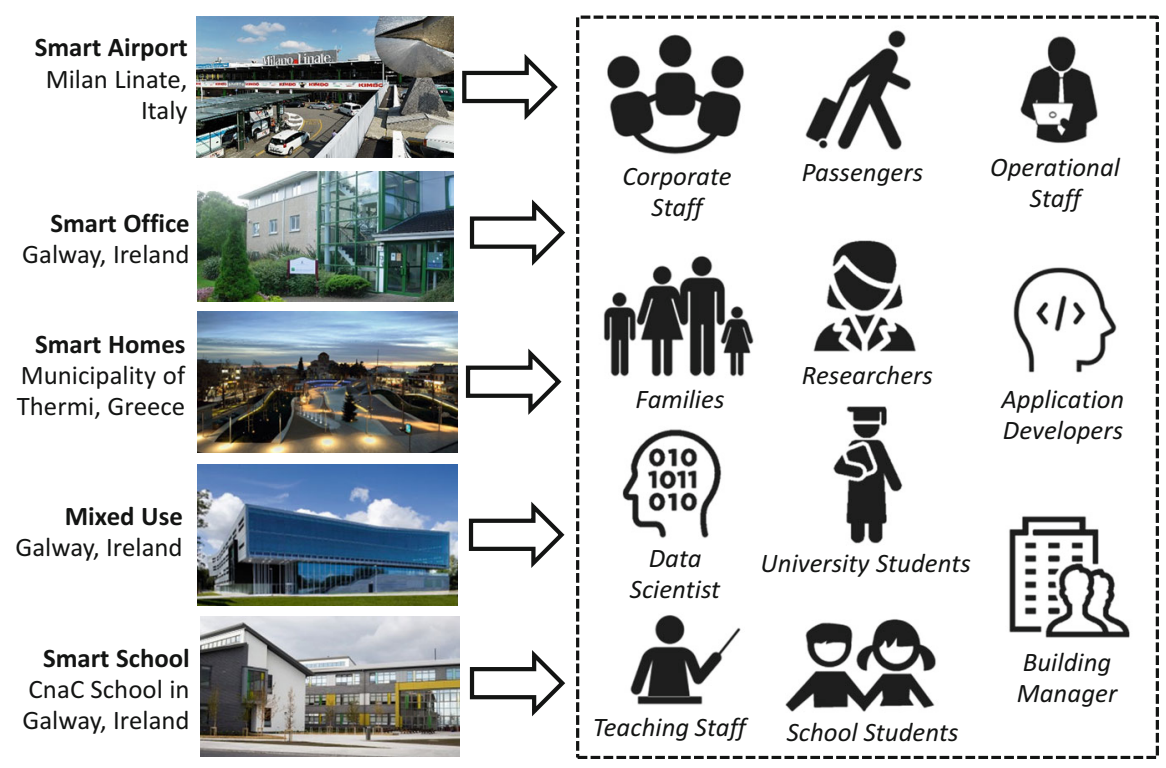

Fig. 14.2 Target user groups across different pilot sites

- Teaching Staff: Teaching staff at both university and school levels looking to use the smart environment as a teaching aid within their classes.

- University Students: Undergraduate and research students are interested in understanding their environment and using the data in their projects and research works. The age groups of this group range from young adults to adults.

- School Students: School students ranging from kids to young adults.

- Data Scientists: Leverage algorithms and machine learning to extract knowledge and insights from data in the smart environment.

- Researchers: Advanced users who want to analyse the data from the smart environment within their discipline specific (i.e. architecture, cognative science) research projects.

- Application Developers: Software and application developers who want to create intelligent applications (including data analytics) for the users within the pilots.

\subsection{Enabling Intelligent Systems, Applications, and Analytics for Smart Environments}

Within a smart environment, data platforms such as Real-time Linked Dataspaces (RLD) [4] are valuable for application developers and data scientists as they constitute a one-stop shop of all the data required for building their intelligent applications or analytics: open data, enterprise data, and sensor data. RLD include public or private data sources that can be used to drive innovations and new solutions within 


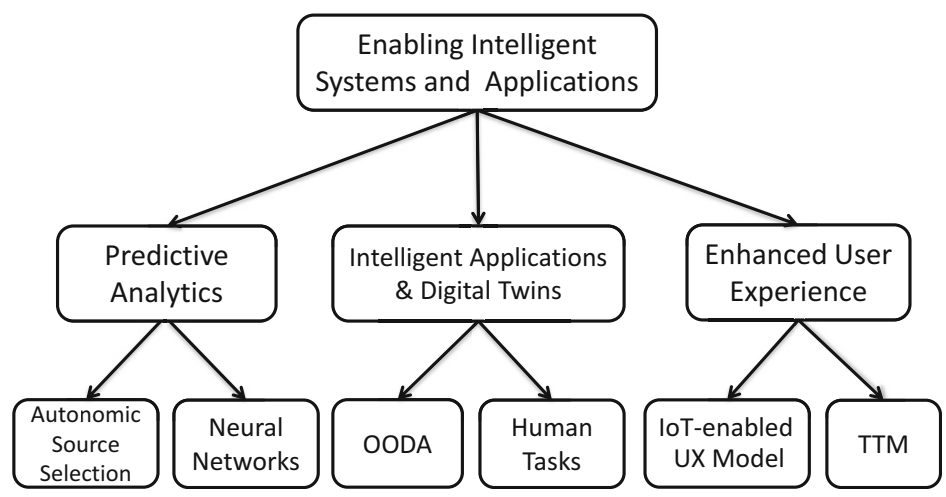

Fig. 14.3 Enabling intelligent systems and applications using the Real-time Linked Dataspace

the smart environment. In this part of the book (see Fig. 14.3), we examine the use of the RLD to provide source selection for real-time predictive analytics, personalised applications for users, and enhanced user experiences for intelligent systems within smart environments.

- Predictive Analytics: A data platform giving access to IoT and open data sources is particularly promising when creating real-time predictive data analytics for decision support within intelligent systems. The main challenge here is the dynamic selection of sources from the dataspace to support predictive analytics. In this context, we are interested in the study of automatic source selection for prediction models in the energy domain using open data and IoT. Chapter 15 presents an autonomic computing inspired approach to source selection for training deep neural networks and machine learning algorithms within the RLD.

- Intelligent Applications and Digital Twins: A key challenge in delivering smart environments is creating effective applications for end-users with new digital infrastructures within the environment. In Chap. 16, we reflect on the experience of using the RLD for developing over 25 different IoT-based intelligent applications and digital twins within five different smart environments, from Airports to Schools. The goal of these has been to engage users within intelligent systems to increase water and energy awareness, management, and conservation. The overall design philosophy of the intelligent applications and digital twins has been guided using Boyd's "OODA Loop” for decision-making.

- User Experience: Creating a compelling user experience within a smart environment (from smart buildings to smart cities) is an essential factor to success. In Chap. 17, we reflect on our experience of developing IoT-based intelligent applications using the RLD where the goal has been to engage a wide range of users (from building managers to business travellers) to increase water and energy awareness, management, and conservation. The design of the experience is defined using an IoT enhanced model for user experience. The user journey within the smart environment is supported by leveraging the Transtheoretical Model of behaviour change to influence a person's attitude positively towards sustainable behaviour. 


\subsection{Summary}

The design of next-generation smart environments poses significant technical challenges with data management, data integration, and real-time processing of dynamic data, and non-technical challenges such as engaging end-users and supporting cultural and organisational changes. Real-time Linked Dataspaces (RLD) are data platforms designed explicitly to tackle these challenges. In this chapter, we provide an overview of how an RLD enables the creation of intelligent systems within a number of smart environments to demonstrate and evaluate their effectiveness to deliver intelligent energy and water management systems. Real-world pilots for smart office, home, school, and airport were introduced together with the different user groups involved in the pilots. Finally, the role of the RLD in enabling intelligent applications for the smart environment from predictive analytics, personalised applications, to enhanced user experience, is introduced.

Open Access This chapter is licensed under the terms of the Creative Commons Attribution 4.0 International License (http://creativecommons.org/licenses/by/4.0/), which permits use, sharing, adaptation, distribution and reproduction in any medium or format, as long as you give appropriate credit to the original author(s) and the source, provide a link to the Creative Commons licence and indicate if changes were made.

The images or other third party material in this chapter are included in the chapter's Creative Commons licence, unless indicated otherwise in a credit line to the material. If material is not included in the chapter's Creative Commons licence and your intended use is not permitted by statutory regulation or exceeds the permitted use, you will need to obtain permission directly from the copyright holder.

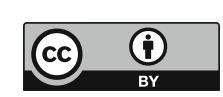

